\title{
DECONVOLUTION WITH WAVELET FOOTPRINTS FOR ILL-POSED INVERSE PROBLEMS
}

\author{
Pier Luigi Dragotti ${ }^{1}$ \\ Martin Vetterli ${ }^{1,2}$ \\ ${ }^{1}$ Laboratoire de Communications Audiovisuelles \\ Swiss Federal Institute of Technology, CH-1015 Lausanne, Switzerland. \\ ${ }^{2}$ EECS Dept., UC Berkeley CA 947020 \\ e-mail: \{pierluigi.dragotti,martin.vetterli\}@epfl.ch \\ http://lcavwww.epfl.ch/ \{dragotti, vetterli\}/
}

\begin{abstract}
In recent years, wavelet based algorithms have been successful in different signal processing tasks. The wavelet transform is a powerful tool, because it manages to efficiently represent sharp discontinuities. Indeed, discontinuities carry most of the signal information and, so, they represent the most critical part to analyse. We have recently introduced the notion of footprints, which form an overcomplete basis built on the wavelet transform. With footprints, one can exactly model the dependency across scales of the wavelet coefficients generated by a discontinuity and this allows to further improve wavelet based algorithms.

In this paper we present a footprint based algorithm for signal deconvolution. The algorithm is fast and works for blind deconvolution too. With footprints we manage to deconvolve efficiently the irregular part of the signal. Thanks to the property of footprints of exactly modeling discontinuities, the deconvolved signal does not present artifacts around discontinuities. Moreover, we show that the residual, that is, the difference between the deconvolved signal with footprints and the observed signal, is regular. Thus, this residual can be further deconvolved with any other traditional method. We show that our system outperforms other deconvolution methods.
\end{abstract}

\section{INTRODUCTION}

Deconvolution is a typical problem that arises in many scientific settings. In its simplest form, the deconvolution problem can be stated as follows. The observed signal $y$ is a degraded version of $x$ and we have:

$$
y[n]=h[n] * x[n]+e[n]
$$

where $h[n]$ is a linear time invariant system and $e[n]$ is an i.i.d. additive Gaussian noise. Given $y$, one aims at estimating $x$, that is, one wants to invert the effect of the filter $h[n]$ and remove the noise. Usually $h[n]$ is known, otherwise it has to be estimated (blind deconvolution). In most cases, $h[n]$ behaves as a low-pass filter and does not have a bounded inverse, for this reason such deconvolution problems are usually called ill-posed.

There is a large number of methods that provide possible solutions to the deconvolution problem $[12,3,9,4,8,1,11,2]$. Some

Pier Luigi Dragotti was supported by Fonds National Suisse project number 20-061493. linear approaches use regularized inverse filters like Wiener filters to invert the effect of $h[n]$. These methods perform well when the signals under consideration are stationary, but in practice this is rarely the case. In particular, many signals of interest (piecewise smooth signals) are characterized by the presence of abrupt non-stationary variations (edges) and, in this case, deconvolution with inverse filters results in signals with evident artifact around the edges. Actually, edges are the part of a signal which is most sensitive to the convolution filter $h[n]$ and, thus, it is the most difficult part to deconvolve. According to the amount of prior information about the signal, one can develop more sophisticated methods which force the estimated signal to meet some prior constraints. Those approaches include methods based on projection onto convex sets and methods based on iterative filters [12, 3, 9]. The main drawback of these techniques is that they are computationally intensive. Finally, wavelet based deconvolution methods have become popular recently $[4,8,1,11,2]$. This is because these methods are inherently non-linear and can deal well with non-stationary signals. Moreover, they are computationally simple.

In this paper, we present a new method based on wavelet transform footprints which further improves wavelet methods. Footprints form an overcomplete basis, which models sharp discontinuities in the wavelet domain well. Footprints form an unconditional basis for piecewise polynomial signals [7]. Thus, if the input signal is piecewise polynomial, the signal enhanced with footprints belongs to the same class as the original one. This allows a reconstruction without artifact around discontinuities. In the more general case of piecewise smooth signals, footprints are well suited to deconvolve the irregular part of that signal. The residual, which we show to be regular, can be efficiently deconvolved with any other method. It is also of interest to highlight that deconvolution with footprints is as simple as a traditional wavelet based denoising algorithm and that it does not require any a-priori knowledge of the filter $h[n]$. Therefore, footprints can be efficiently used for blind deconvolution.

In the next two sections, we describe the class of signals we are interested in, namely piecewise smooth signals and present the footprint expansion. In Section 4, we introduce our deconvolution algorithm based on footprints and in Section 5 we show some numerical results. We conclude in Section 6. 


\section{SIGNALS MODELS}

In this work, we consider piecewise smooth signals, that is, signals which are made of smooth pieces. For example, we define a piecewise smooth function $f(t), t \in[0, T]$ with $K+1$ pieces, as follows:

$$
f(t)=\sum_{i=0}^{K} f_{i}(t) \mathbf{1}_{\left[t_{i}, t_{i+1}\right]}(t)
$$

where $t_{0}=0, t_{K+1}=T$ and $f_{i}(t)$ is uniformly Lipschitz $\alpha$ over $[0, T]^{1}$. Those signals are interesting, because many signals encountered in practice can be well modeled as piecewise smooth. There is also another set of functions we will consider and which form the more restricted class of piecewise polynomial signals. A function $p(t) t \in[0, T]$ is piecewise polynomial with $K+1$ pieces if:

$$
p(t)=\sum_{i=0}^{K} p_{i}(t) \mathbf{1}_{\left[t_{i}, t_{i+1}\right]}(t),
$$

where $t_{0}=0, t_{K+1}=T$ and $p_{i}(t), \quad i=0,1, . . K$ are polynomials of maximum degree $D$.

Despite their simplicity, piecewise polynomial signals represent an important tool to characterize the non-stationary behaviour of piecewise smooth functions. It follows [7]:

Theorem 1 Given a piecewise smooth signal $f(t)$ defined as in $E q$. (2). There always exists a piecewise polynomial signal $p(t)$ with pieces of maximum degree $p=\lfloor\alpha\rfloor$ such that the difference signal $g(t)=f(t)-p(t)$ is uniformly Lipschitz $\alpha^{\prime}\left(p<\alpha^{\prime} \leq \alpha\right)$ over $[0, T]$.

This theorem shows that one can separate any piecewise smooth signal in a piecewise polynomial part and a residual which is regular. Now, we will show in the next section, that footprints give a sparse and exact representation of piecewise polynomial signals. This is why our deconvolution algorithm operates in two steps. First, we use footprints to estimate the correct piecewise polynomial behaviour underlying the corrupted piecewise smooth signal. Then we use a traditional approach like Wiener filtering to enhance the residual, which Theorem 1 has shown to be regular. Doing so, we are very efficient in enhancing the sharp discontinuities of the signal. While, other techniques usually fail to enhance discontinuities well.

\section{THE FOOTPRINT EXPANSION}

We move, now, from continuous time to discrete-time signals. In [6], we have introduced the notion of footprints and proposed their use for compression and denoising. What is interesting is that footprints can be seen as an overcomplete basis for representation of piecewise polynomial functions [7].

Consider, first, a piecewise constant signal $x[n], n \in[0, N-$ $1]$ with only one discontinuity at position $k$. Consider a $J$ level wavelet decomposition of this signal with a Haar wavelet:

$$
x[n]=\sum_{j=1}^{J} \sum_{l=0}^{N / 2^{j}-1} y_{j l} \psi_{j l}[n]+\sum_{l=0}^{N / 2^{J}-1} c_{l} \phi_{J l}[n] .
$$

\footnotetext{
${ }^{1}$ For a definition of Lipschitz regularity refer to [10].
}

where $y_{j l}=\left\langle x, \psi_{j l}\right\rangle$, and $c_{l}=\left\langle x, \phi_{J l}\right\rangle^{2}$. Now, since the Haar wavelet has one vanishing moment and finite support, the non-zero wavelet coefficients of this decomposition are only in the cone of influence of $k$. Thus Eq. (4) becomes:

$$
x[n]=\sum_{j=1}^{J} y_{j k_{j}} \psi_{j k_{j}}[n]+\sum_{l=0}^{N / 2^{J}-1} c_{l} \phi_{J l}[n],
$$

where $k_{j}=\left\lfloor k / 2^{j}\right\rfloor-1$. Moreover, all those coefficients depend only on the amplitude of the discontinuity at $k$. Thus, if one defines a vector which contains all of them, one can specify any other step discontinuity at $k$ by multiplying this vector by the right factor. This consideration leads to the following definition:

Definition 1 Given a piecewise constant signal $x$ with only one discontinuity at position $k$, we call footprint $f_{k}^{(0)}$ the norm one scale-space vector obtained by gathering together all the wavelet coefficients in the cone of influence of $k$ and then imposing $\left\|f_{k}^{(0)}\right\|=$ 1.

Expressed in the wavelet basis, this footprint can be written as $f_{k}^{(0)}[n]=\sum_{j=1}^{J} d_{j k_{j}} \psi_{j k_{j}}[n]$, where $d_{j k_{j}}=y_{j k_{j}} / \sqrt{\sum_{j=1}^{J} y_{j k_{j}}^{2}}$. Now, any piecewise constant signal $x[n]$ with a step discontinuity at $k$ can be represented in terms of the scaling functions $\phi_{J l}[n]$ and of $f_{k}^{(0)}$. For instance, the signal $x[n]$ in Eq. (4) becomes:

$$
x[n]=\sum_{l=0}^{N / 2^{J}-1} c_{l} \phi_{J l}[n]+\alpha f_{k}^{(0)}[n],
$$

where $\alpha=<x, f_{k}^{(0)}>=\sum_{j=0}^{J} y_{j k_{j}} d_{j k_{j}}$. That is, $\left\langle x, f_{k}^{(0)}>\right.$ represents the inner product between $f_{k}^{(0)}$ and the wavelet coefficients of $x$ located at the same scale-space positions of the coefficients of $f_{k}^{(0)}$. The above discussion can be repeated for any other step discontinuity at different locations. For each location $l$ we have a different footprint $f_{l}^{(0)}$. Now, given the complete dictionary $\mathcal{D}=\left\{f_{k}^{(0)}, k=1,2, . . N-1.\right\}$ of footprints, we can express any piecewise constant signal in terms of the elements of this dictionary and of the scaling functions.

The notion of footprints can be easily generalized to the case of piecewise polynomial signals (for more details refer to [7]). In this case, it can be shown that the wavelet coefficients in the cone of influence of a polynomial discontinuity at location $k$ have only $D+1$ degrees of freedom ( $D$ is the maximum degree of any polynomial in the signal). Thus, one can characterize this discontinuity using $D+1$ footprints $f_{k}^{(d)}, d=0,1, . . D$.. To characterize any polynomial discontinuity, we need a dictionary $\mathcal{D}=\left\{f_{k}^{(d)}, d=0,1, . ., D ; k=0,1, . ., N-1.\right\}$ of $(D+1) N$ footprints. With this dictionary of footprints and with the scaling functions, we can represent any piecewise polynomial signal. In particular, a piecewise polynomial signal $x$ with $K$ discontinuities at locations $k_{1}, k_{2}, . . k_{K}$ is given by:

$$
x[n]=\sum_{l=0}^{N / 2^{J}-1} c_{l} \phi_{J l}[n]+\sum_{i=0}^{K} \sum_{d=1}^{D} \alpha_{i}^{(d)} f_{k_{i}}^{(d)}[n] .
$$

Footprints are orthogonal to the scaling functions. Footprints related to the same locations are orthogonal too (i.e. $\left\langle f_{k}^{(c)}, f_{k}^{(d)}>=\right.$

\footnotetext{
${ }^{2}$ Note that we are assuming $N$ to be a power of 2 .
} 
$\left.\delta_{c d}\right)$. But footprints related to close discontinuities are biorthogonal. In particular, we have: $\left\langle f_{l}^{(d)}, f_{k}^{(c)}\right\rangle=0$ for $|l-k|>$ $(L-1) \cdot 2^{J}$, where $L$ is the length of the wavelet filter. Thus, the orthogonality of footprints depend on the number $J$ of wavelet decomposition level. Now, assume that we know the discontinuity locations and call $k_{m-1}, k_{m}$ the two closest discontinuities in (5). If $J$ is chosen such that $J=\left\lfloor\log _{2}\left(k_{m}-k_{m-1}\right)-\log _{2}(L-1)\right\rfloor$, than we are sure that the footprints related to locations $k_{1}, k_{2}, . ., k_{K}$ are orthogonal. In the next section, we present an iterative algorithm where the number $J$ is chosen adaptively according to the distance between discontinuities. In this way, there are no biorthogonal footprints to represent that signal. It is also of interest to note that footprints manage to give a sparser representation of piecewise polynomial signals than the wavelet transform. Moreover, when $J=\log _{2} N$, footprints form an unconditional basis for piecewise polynomial signals. That is, any linear combination of footprints gives a signal which is piecewise polynomial.

\section{DECONVOLUTION WITH FOOTPRINTS}

Assume that the observed signal $y[n]$ is given by eq. (1) and that the original signal $x[n]$ is piecewise polynomial. Moreover, call $\sigma^{2}$ the variance of the Gaussian noise $e[n]$. Now, piecewise polynomial signals can be represented with footprints, thus our deconvolution algorithm simply try to estimate the footprint representation of $x[n]$ from the observed version $y[n]$.

For simplicity let us focus on piecewise constant signals. We first perform an estimation of the discontinuity locations and then we estimate the values of the footprints coefficients $\alpha_{i}^{(0)}$. The discontinuity locations are estimated in the following way:

1. Chose a dictionary $\mathcal{D}=\left\{f_{k}^{(0)}=\sum_{j=1}^{J} d_{j k_{j}} \psi_{j k_{j}} ; k=\right.$ $0,1, . . N-1\}$ of footprints with $J=\log _{2} N$. This dictionary represents a biorthogonal basis.

2. Compute the dual basis of $\mathcal{D}$ and call $\tilde{f}_{k}^{(0)} k=1,2, . ., N-$ 1 the elements of this dual basis ${ }^{3}$.

3. Compute the $N-1$ inner products $\left\langle y, \tilde{f}_{k}^{(0)}>k=\right.$ $1,2, . ., N-1$.

4. Consider as discontinuity locations the ones related to the inner products larger than the threshold $T_{k}=\left\|\tilde{f}_{k}^{(0)}\right\| T$. That is, if $\left|<y, \tilde{f}_{k}^{(0)}>\right| \geq T_{k}$, then assume that there is a discontinuity at location $k . T$ is the universal threshold $(T=\sigma \sqrt{(2 \ln N)})$ [5]

Now, we have a set of estimated discontinuity locations: $\hat{k}_{1}, \hat{k}_{2}, . ., \hat{k}_{\hat{K}}$. The problem is that, due to the noise, this estimation can have errors. Thus, this problem must be considered in the next step where the footprints coefficients are evaluated.

1. Given the set of estimated discontinuity locations, take $J_{1}=$ $\left\lfloor\log _{2}\left(\hat{k}_{m}-\hat{k}_{m-1}\right)-\log _{2}(L-1)\right\rfloor$, where $\hat{k}_{m-1}, \hat{k}_{m}$ are the two closest estimated discontinuity locations.

2. For each possible location $k \in\left[\hat{k}_{m-1}, \hat{k}_{m}\right]$ compute the inner product $\left\langle y, \frac{\hat{f}_{k}^{(0)}}{\left\|\hat{f}_{k}^{(0)}\right\|}>\right.$, where $\hat{f}_{k}^{(0)}$ is the sub-footprint obtained by considering only the first $J_{1}$ wavelet coefficients of $f_{k}^{(0)}$. That is: $\hat{f}_{k}^{(0)}=\sum_{j=1}^{J_{1}} d_{j k_{j}} \psi_{j k_{j}}[n]$

${ }^{3}$ It is of interest to emphasize that this dual basis turns out to be a first order derivative.
3. Choose the location $k_{1}$ such that $\left|<y, \frac{\hat{f}_{k_{1}}^{(0)}}{\left\|\hat{f}_{k_{1}}^{(0)}\right\|}>\right|$ is maximum.

4. If

$$
\left|<y, \frac{\hat{f}_{k_{1}}^{(0)}}{\left\|\hat{f}_{k_{1}}^{(0)}\right\|}>\right| \geq T
$$

then compute the residue:

$$
R_{y}^{1}=y-\frac{1}{\left\|\hat{f}_{k_{1}}^{(0)}\right\|}<y, \frac{\hat{f}_{k_{1}}^{(0)}}{\left\|\hat{f}_{k_{1}}^{(0)}\right\|}>f_{k_{1}}^{(0)} .
$$

5. Iterate step 3-4 on the residue until condition (6) is not verified anymore.

6. Once condition (6) is not verified anymore, remove the two discontinuity locations $\hat{k}_{j-1}, \hat{k}_{j}$. If the set of remaining discontinuity locations is not empty, find a new decomposition level $J_{2}$ and go to step 2. Otherwise, if all discontinuities have been considered, stop.

Finally, the estimated signal $\hat{x}$ is:

$$
\hat{x}=<y, \phi_{J}>\phi_{J}[n]+\sum_{m=0}^{M-1} \frac{1}{\left\|\hat{f}_{k_{m}}^{(0)}\right\|}<R_{y}^{m}, \frac{\hat{f}_{k_{m}}^{(0)}}{\left\|\hat{f}_{k_{m}}^{(0)}\right\|}>f_{k_{m}}^{(0)}[n],
$$

where $M$ is the total number of iterations and $R_{y}^{0}=y$.

First, notice that, since the footprints $f_{k_{m}}^{(0)}$ in Eq. (7) are obtained taking a wavelet transform with $J=\log _{2} N$ decomposition level, then we are sure that the estimated signal $\hat{x}$ is piecewise constant as $x$. This is an important property, because in this way we are sure that the estimated signal does not present artifact around discontinuities. This algorithm can be easily generalized to the case of piecewise polynomial signals and, thus, we do not detail this generalization here.

Now, assume that the original signal $x[n]$ is piecewise smooth. In this case, we use a two step deconvolution algorithm. First, we estimate the piecewise polynomial behaviour of $x$ using this footprints based algorithm. Then we use a Wiener filter to deconvolve the residual $r[n]=y[n]-\hat{x}[n]$.

\section{SIMULATION RESULTS}

To analyse the performance of our system, we consider two different signals. One is the classical 'Blocks' signal which is an example of piecewise constant signal, the other is one line of the image 'Cameraman', which represents a possible example of piecewise smooth signal. In Figure 5, we show the performance of our system for the 'Blocks' signal and compare it with WaRD [11]. In this simulation, the original signal is first convolved with a Gaussian filter and then white noise is added. The noise variance is set to $\sigma^{2}=0.2$. We can see that our system outperforms WaRD system in both visual quality and SNR. In particular, the signal reconstructed with footprints does not present artifacts around discontinuities.

In Figure 5, we consider the case where the signal is piecewise smooth. Again, the original signal is convolved with a Gaussian filter and then white noise is added. In this case we use the two 
(a)

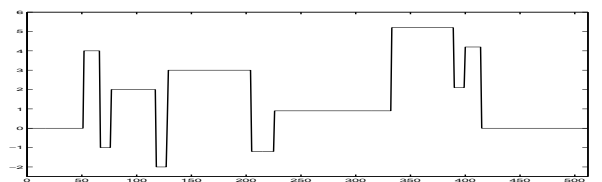

(b)
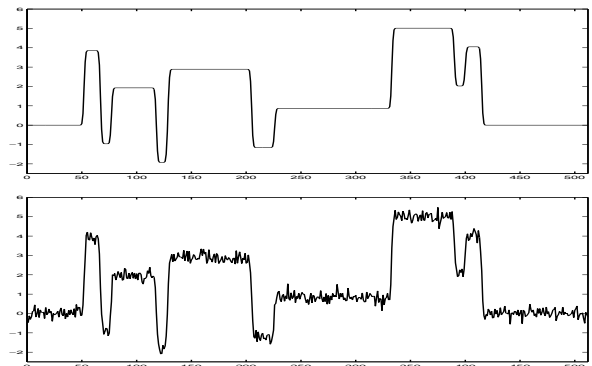

(c)

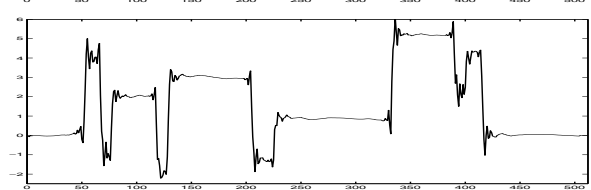

(d)

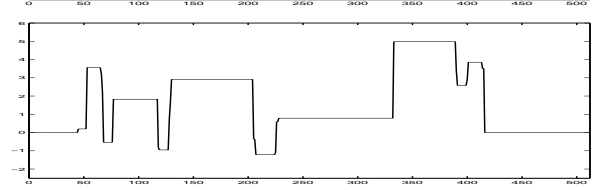

(a)

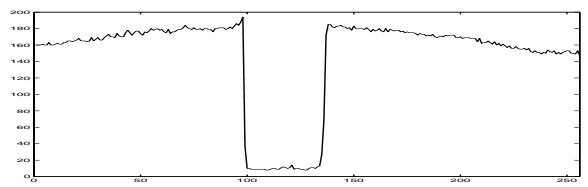

(b)

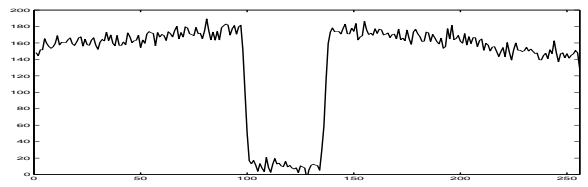

(c)

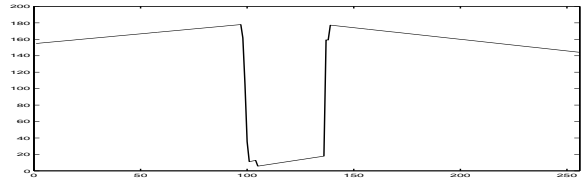

(d)

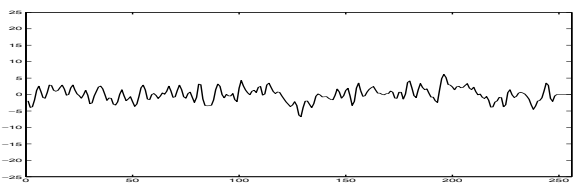

(e)

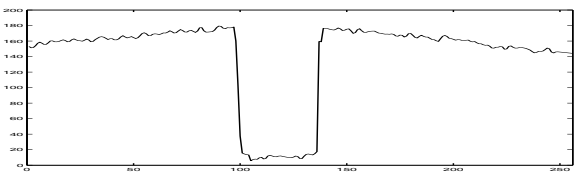

Fig. 1. (a) Test signal $(\mathrm{N}=512)$. (b) Signal convolved with a Gaussian filter. (c) Observed signal. (d) Deconvolution with Ward $(\mathrm{SNR}=15.2 \mathrm{~dB})$. (e) Deconvolution with Footprints $(\mathrm{SNR}=16.4 \mathrm{~dB})$.

steps algorithm proposed in the previous section. We, first, estimate the piecewise polynomial behaviour underlying the signal (c) and then we deconvolve the residual with a Wiener filter (d). The reconstructed signal is shown in Fig. 5 (e).

\section{CONCLUSIONS}

In this paper we have proposed a new deconvolution algorithm based on footprints. The two main feature of this method are its simplicity and its effectiveness in dealing with sharp discontinuities. The generalization of this algorithm to the case of 2dimensional signal is a topic under investigation.

\section{REFERENCES}

[1] M.R. Banham and A.K. Katsaggelos, "Spatially adaptive wavelet-based multiscale image restoration," IEEE Trans. Image Processing, vol.5, pp. 619-634, April 1996.

[2] K. Berkner, M. Gormish and E. Schwartz, "Multiscale Sharpening and Smoothing in Besov Spaces with Applications to Image Enhancement," App. Comp. Harmonic Anal., vol.11, pp. 2-31, 2001.

[3] P.L. Combettes, "Generalized Convex Set Theoretic Image Recovery,” Int. Conf. on Image Processing, 1996.

[4] D.L.Donoho, "Nonlinear solution of linear inverse problems by Wavelet-Vaguelette Decomposition," App. Comp. Harmonic Anal., vol.2, pp. 101-126, 1995.
Fig. 2. (a) Test signal ( $\mathrm{N}=256)$. (b) Observed signal. (c) Piecewise polynomial estimation. (d) Deconvolution of the residual with a Wiener filter. (e) Complete deconvolved signal ( $\mathrm{SNR}=25 \mathrm{~dB})$.

[5] D.L.Donoho and I.M. Johnstone, "Ideal spatial adaptation via wavelet shrinkage,” Biometrika, 81:425-455, 1994.

[6] P.L. Dragotti and M. Vetterli, "Wavelet transform footprints: catching singularities for compression and denoising”, Int. Conf. on Image Processing, Vancouver, 2000.

[7] P.L. Dragotti and M. Vetterli, "The Overcomplete Footprint Expansion," to be submitted.

[8] J. Kalifa, S. Mallat and B. Rouge, "Image deconvolution in mirror wavelet bases," Int. Conf. on Image Processing, Chicago, 1998.

[9] A.K. Katsaggelos, "Iterative image restoration algorithm," Opt. Eng, vol. 28, 735-748, 1989.

[10] S. Mallat, A Wavelet Tour of Signal Processing, Academic Press, 1998.

[11] R. Neelamani, Hyeokho Choi and Richard Baraniuk "Wavelet-Based Deconvolution for Ill-Conditioned Systems," Int. Conf. Acoustic, Speech and Signal Proc., 1999.

[12] H. Stark, Image Recovery: Theory and Application, Academic Press, 1987. 